TRANSACTIONS OF THE

AMERICAN MATHEMATICAL SOCIETY

Volume 351, Number 9, Pages 3713-3724

S 0002-9947(99)02249-7

Article electronically published on March 1, 1999

\title{
A GLOBAL CONDITION FOR PERIODIC DUFFING-LIKE EQUATIONS
}

PIERO MONTECCHIARI, MARGHERITA NOLASCO, AND SUSANNA TERRACINI

Abstract. We study Duffing-like equations of the type $\ddot{q}=q-\alpha(t) W^{\prime}(q)$, with $\alpha \in C(\mathbb{R}, \mathbb{R})$ periodic. We prove that if the stable and unstable manifolds to the origin do not coincide, then the system exhibits positive topological entropy.

\section{INTRODUCTION}

In this paper we consider the class of Duffing-like equations

$$
\ddot{x}=x-\alpha(t) W^{\prime}(x), \quad(t, x) \in \mathbb{R} \times \mathbb{R},
$$

where we assume:

(h1) $\alpha \in C(\mathbb{R})$ positive and T-periodic;

(h2) $W \in C^{2}(\mathbb{R})$ and $W(0)=W^{\prime}(0)=W^{\prime \prime}(0)=0$;

(h3) there exists $\beta>2$ such that $0<\beta W(x) \leq W^{\prime}(x) \cdot x$ for any $x \neq 0$;

(h4) $W^{\prime}(x) \cdot x<W^{\prime \prime}(x) x \cdot x$ for any $x \neq 0$;

(h5) $W(x)=W(-x)$ for any $x \in \mathbb{R}$.

We can take $W(x)=|x|^{\beta}$ with $\beta>2$ as model potentials. Under the above assumptions, system (1.1) has the origin of the phase plane as a hyperbolic rest point; a solution is called homoclinic to the origin if $x(t) \rightarrow 0$ and $\dot{x}(t) \rightarrow 0$ as $t \rightarrow \pm \infty$.

Starting from [3] and [7] the existence of homoclinic motions for periodic Hamiltonian systems has been studied by variational methods. Using these techniques, E. Séré ([17] and [18]) obtained the first multiplicity results for first-order convex and superquadratic Hamiltonian systems, periodic in time. Analogous results have been obtained in [8] for second order systems (we mention [11] and the references therein for more general time dependences). The proof of these results involves the construction of a class of solutions, called multibump solutions, and reveals some chaotic features of the dynamics (the presence of an approximate Bernoulli shift and the positive topological entropy). The main requirement for this construction is that

$(*) \quad$ the set of homoclinic solutions is countable.

This condition generalizes to a certain extent other non-degeneracy conditions that have been used in the Dynamical System Theory in order to detect a chaotic behaviour. It is worthwhile noticing that $(*)$ is verified if the stable and unstable

Received by the editors July 16, 1996 and, in revised form, March 31, 1997.

1991 Mathematics Subject Classification. Primary 58E05, 70H35, 34C37, 58F15.

Key words and phrases. Duffing equations, homoclinic orbits, multibump solutions, minimax argument. 
manifolds to the origin intersect transversally, that is the key assumption in the classical Smale-Birkhoff theorem (see e.g. [19], [12], [20]). Moreover we mention the notion of exponential dichotomy used in [14] in proving a shadowing lemma (see also [5] for a first study of Duffing-like equations).

In this paper, using variational methods, we prove that a shadowing-like lemma holds for (1.1) whenever the stable and unstable manifolds to the origin do not coincide (see [2], [4], [6], [16] for others results in this direction).

Let us recall the notions of stable and unstable manifolds. Since the system is $T$ periodic we can consider the $T$-map $\Phi: \mathbb{R}^{2} \rightarrow \mathbb{R}^{2}$, given by $\Phi(x, p)=(u(T), \dot{u}(T))$, where $u$ is the solution of (1.1) with initial conditions $u(0)=x$ and $\dot{u}(0)=p$. Then, we can define the stable and unstable manifolds to the hyperbolic rest point $(x, p)=(0,0)$ as follows:

$$
\begin{array}{ll}
\mathcal{W}^{s}=\left\{(x, p) \in \mathbb{R}^{2}: \Phi^{n}(x, p) \rightarrow 0,\right. & \text { as } n \rightarrow+\infty\}, \\
\mathcal{W}^{u}=\left\{(x, p) \in \mathbb{R}^{2}: \Phi^{n}(x, p) \rightarrow 0,\right. & \text { as } n \rightarrow-\infty\} .
\end{array}
$$

Note that $\mathcal{W}^{s} \cap \mathcal{W}^{u} \backslash\{0\}$ is the set of initial data of homoclinic solutions.

We prove

Theorem 1.1. If $\mathcal{W}^{u} \neq \mathcal{W}^{s}$ then (1.1) admits multibump-type solutions. Precisely, there exists a set $K$ of homoclinic solutions of (1.1) that is compact in $C^{1}(\mathbb{R})$ and such that $0 \notin K$, for which, for any $r>0$ there is $N_{r}>0$ such that for any sequence $\left(p_{j}\right) \subset \mathbb{Z}$, with $p_{j+1}-p_{j} \geq N_{r}$, and $\sigma=\left(\sigma_{j}\right) \subset\{0,1\}^{\mathbb{Z}}$, there exists a solution $v_{\sigma}$ of (1.1) which verifies

$$
\inf _{u \in K}\left\|v_{\sigma}-\sigma_{j} u\left(\cdot-p_{j} T\right)\right\|_{C^{1}\left(I_{j}\right)}<r,
$$

for any $j \in \mathbb{Z}$, where $I_{j}=\left[\frac{p_{j-1}+p_{j}}{2} T, \frac{p_{j}+p_{j+1}}{2} T\right]$. In addition $v_{\sigma}$ is a homoclinic orbit whenever $\sigma_{j}=0$, definitively.

The presence of a multibump dynamics implies in particular that the set of homoclinic solutions cannot be compact up to traslations in $\mathcal{C}^{1}(\mathbb{R} ; \mathbb{R})$. Hence the assumption $\mathcal{W}^{s} \neq \mathcal{W}^{u}$ is clearly a necessary condition for the theorem. Otherwise, as a subset of $\mathcal{C}^{1}(\mathbb{R} ; \mathbb{R})$, the set of homoclinics is a one-dimensional manifold, compact up to $T$-translations.

The assertion of the theorem can be read as the existence of an approximate (discontinuous) Bernoulli shift. This implies in particular that if $\mathcal{W}^{s} \neq \mathcal{W}^{u}$ the system exhibits sensitive dependence on initial data. In fact, a stronger property holds. Let us introduce the following definition of topological entropy (see e.g. $[15])$,

$$
h(\Phi)=\sup _{R>0} \lim _{\epsilon \rightarrow 0} \limsup _{n \rightarrow \infty} \frac{1}{n} \log s(n, \epsilon, R)
$$

with $s(n, \epsilon, R)=\max \left\{\operatorname{card}(E) ; E \subset B_{R}(0), \max _{0 \leq k \leq n}\left|\Phi^{k}(x)-\Phi^{k}(y)\right| \geq \epsilon, \forall x \neq\right.$ $y \in E\}$.

Then, if $\mathcal{W}^{s} \neq \mathcal{W}^{u}$ the topological entropy of (1.1) is positive, i.e., the number of points which are separated by the discrete flow in $n$-iterations increases exponentially with $n$.

Indeed, for any $u \in K$, let $x_{u}=(u(0), \dot{u}(0)) \in \mathbb{R}^{2}$. Fix $r \leq \inf _{u \in K} \frac{1}{3}\left|x_{u}\right|$ and a sequence $\left(p_{k}\right) \subset \mathbb{Z}$ such that $p_{k}=k N_{r},(\forall k \in \mathbb{Z})$, where $K \subset C^{1}(\mathbb{R})$ and $N_{r} \in \mathbb{N}$ are given by the above theorem. Let $\sigma, \sigma^{\prime} \subset\{0,1\}^{\mathbb{Z}}$ such that $\sigma_{j} \neq \sigma_{j}^{\prime}$ for some 
$j \in \mathbb{Z}$ and $v_{\sigma}, v_{\sigma^{\prime}}$ the corresponding solutions of (1.1). Since $\sigma_{j} \neq \sigma_{j}^{\prime} \in\{0,1\}$, setting $\tau(\sigma)=\left(v_{\sigma}(0), \dot{v}_{\sigma}(0)\right)$, we have

$$
\begin{aligned}
\left|\Phi^{j N_{r}}(\tau(\sigma))-\Phi^{j N_{r}}\left(\tau\left(\sigma^{\prime}\right)\right)\right| & \geq \inf _{u \in K}\left|x_{u}\right|-\inf _{u \in K}\left(\left|\Phi^{j N_{r}}(\tau(\sigma))-\sigma_{j} x_{u}\right|\right. \\
& \left.\left.+\left|\Phi^{j N_{r}}\left(\tau\left(\sigma^{\prime}\right)\right)-\sigma_{j}^{\prime} x_{u}\right|\right)\right) \\
& \geq \inf _{u \in K}\left|x_{u}\right|-\left(\inf _{u \in K}\left\|v_{\sigma}-\sigma_{j} u\left(\cdot-p_{j} T\right)\right\|_{C^{1}\left(I_{j}\right)}\right. \\
& \left.+\inf _{u \in K}\left\|v_{\sigma^{\prime}}-\sigma_{j}^{\prime} u\left(\cdot-p_{j} T\right)\right\|_{C^{1}\left(I_{j}\right)}\right) \geq \inf _{u \in K} \frac{1}{3}\left|x_{u}\right|
\end{aligned}
$$

Hence, for $\epsilon \leq \inf _{u \in K} \frac{1}{3}\left|x_{u}\right|$ and $R \geq \sup _{u \in K}\left|x_{u}\right|+r$, we get $s\left(n N_{r}, \epsilon, R\right) \geq 2^{n}$ and $h(\Phi) \geq \frac{\log 2}{N_{r}}$.

The paper is organized as follows: in section 2 we introduce the variational setting and we recall some preliminary results. In section 3 we study, from the variational point of view, the consequences of the geometrical assumption $\mathcal{W}^{s} \neq \mathcal{W}^{u}$. In particular, we show how this condition allows us to use the multibump construction developed in [18], which we apply in the last section to prove our result.

In the following we will assume $T=1$ and we will denote by $C$ a positive constant which may be different from time to time.

Acknowledgement. We wish to thank V. Coti Zelati and E. Séré for useful comments and suggestions.

\section{Preliminary Results}

We look for homoclinic solutions of (1.1) as critical points of the action functional $\varphi(u)=\frac{1}{2}\|u\|^{2}-\int_{\mathbb{R}} \alpha(t) W(u)$, defined on the Sobolev space $X=H^{1}(\mathbb{R})$ endowed with the standard euclidean norm $\|u\|^{2}=\int_{\mathbb{R}}|\dot{u}|^{2}+|u|^{2} d t$.

By (h1) and (h2), $\varphi \in C^{2}(X, \mathbb{R})$ and $\varphi^{\prime}(u) h=\langle u, h\rangle-\int_{\mathbb{R}} \alpha(t) W^{\prime}(u) h$, for any $u, h \in X$. Let us denote $\mathcal{K}=\left\{v \in X \backslash\{0\}: \varphi^{\prime}(v)=0\right\}$.

We note that by (h1) and (h2) the origin in $X$ is a strict local minimum for $\varphi$. Indeed, we can fix $\bar{\delta}>0$ such that

$$
\left|\alpha(t) W^{\prime \prime}(x)\right| \leq \frac{1}{4} \quad \forall t \in \mathbb{R} \text { and }|x| \leq \bar{\delta} .
$$

Since $W(x)=\int_{0}^{1} W^{\prime}(s x) \cdot x d s$ and $W^{\prime}(x)=\int_{0}^{1} W^{\prime \prime}(s x) \cdot x d s$, we have $|\alpha(t) W(x)| \leq$ $\frac{1}{8}|x|^{2}$ and $\left|\alpha(t) W^{\prime}(x)\right| \leq \frac{1}{4}|x|$ for any $|x| \leq \bar{\delta}, t \in \mathbb{R}$.

By the Sobolev imbedding theorem there exists $M>0$ such that if $I \subset \mathbb{R}$ is an interval with $|I| \geq 1(|I|$ denotes the measure of $I)$, then

$$
\sup _{t \in I}|u(t)| \leq M\|u\|_{I}
$$

where $\|u\|_{I}^{2}=\int_{I}|\dot{u}(t)|^{2}+|u(t)|^{2} d t, u \in X$. Then there exists $\bar{\rho}>0$ such that

$$
\|u\|_{I} \leq \bar{\rho} \Rightarrow \sup _{t \in I}|u(t)| \leq \bar{\delta}
$$

We have

Lemma 2.1. $\varphi(0)=0$ and if $\|u\|_{\infty} \leq \bar{\delta}$, then $\varphi(u) \geq \frac{1}{4}\|u\|^{2}$ and $\varphi^{\prime}(u) u \geq \frac{1}{2}\|u\|^{2}$. In particular $\inf _{v \in \mathcal{K}}\|v\|_{\infty}>\bar{\delta}$. 
By (h3) we get that $W(s x) \geq C s^{\beta}$ as $s \rightarrow \infty$ for any $|x|=1$. Therefore, since $\beta>2$, we obtain that if $u \neq 0$, then $\varphi(\lambda u) \rightarrow-\infty$ as $\lambda \rightarrow \infty$.

We introduce the class of paths

$$
\Gamma=\{\gamma \in C([0,1], X): \gamma(0)=0, \varphi(\gamma(1))<0\}
$$

and we define $c=\inf _{\gamma \in \Gamma} \max _{s \in[0,1]} \varphi(\gamma(s))$. By the mountain pass theorem $c>0$ and there exists a Palais Smale (PS) sequence at level $c$, i.e., a sequence $\left(u_{n}\right) \subset X$ such that $\varphi\left(u_{n}\right) \rightarrow c$ and $\varphi^{\prime}\left(u_{n}\right) \rightarrow 0$.

By (h3) we have also

$$
\left(\frac{1}{2}-\frac{1}{\beta}\right)\|u\|^{2} \leq \varphi(u)+\frac{1}{\beta}\left|\varphi^{\prime}(u) u\right| \quad \forall u \in X
$$

from which we derive that the PS sequences are actually bounded sequences.

By periodicity, if $\left(u_{n}\right) \subset X$ is a PS sequence, then for any sequence $\left(t_{n}\right) \subset \mathbb{Z}$, $\left(u_{n}\left(\cdot-t_{n}\right)\right)$ is still a PS sequence. Hence, in particular, the Palais Smale condition does not hold. The asymptotic behaviour of PS sequences can be characterized by concentration compactness arguments [9]. We have (see e.g. [8] for a proof):

Lemma 2.2. Let $\left(u_{n}\right) \subset X$ be a PS sequence for $\varphi$ at the level $b>0, u_{n} \rightarrow$ $v$ weakly in $X$. Then $v \in \mathcal{K} \cup\{0\}$ and there exist $k \in \mathbb{N} \cup\{0\}, k$ sequences $\left(t_{n}^{1}\right), \ldots,\left(t_{n}^{k}\right) \subset \mathbb{Z}$ and $k$ critical points $v_{1}, \ldots, v_{k} \in \mathcal{K}$ such that, as $n \rightarrow \infty$, up to subsequences,

1. $\left|t_{n}^{i}\right| \rightarrow \infty$ and $\left|t_{n}^{i}-t_{n}^{j}\right| \rightarrow \infty$ as $n \rightarrow \infty$ whenever $i \neq j$;

2. $\left\|u_{n}-v-\sum_{i=1}^{k} v_{i}\left(\cdot-t_{n}^{i}\right)\right\| \rightarrow 0$ as $n \rightarrow \infty$;

3. $b=\varphi(v)+\sum_{i=1}^{k} \varphi\left(v_{i}\right)$.

By lemmas 2.1 and 2.2 we obtain a local compactness property.

Lemma 2.3. If $K$ is a compact subset of $X$ and $\left(u_{n}\right) \subset X$ is a PS sequence of $\varphi$ such that $d\left(u_{n}, K\right) \leq \bar{\rho}$, then $\left(u_{n}\right)$ is precompact in $X$.

By (h4), the mountain pass level $c$ has the following property:

Lemma 2.4. $\inf \{\varphi(u): u \in \mathcal{K}\} \geq c$.

Proof. If $u \in \mathcal{K}$ we have that $\|u\|^{2}=\int_{\mathbb{R}} \alpha(t) W^{\prime}(u) u d t$. Therefore for $s \in \mathbb{R}$

$$
\frac{d}{d s} \varphi(s u)=s\|u\|^{2}-\int_{\mathbb{R}} \alpha(t) W^{\prime}(s u) u=s \int_{\mathbb{R}} \alpha(t)\left(W^{\prime}(u) u-\frac{1}{s} W^{\prime}(s u) u\right) d t .
$$

By (h4), for any $x \in \mathbb{R}^{\mathbb{N}} \backslash\{0\}$ the function $f_{x}(s)=\frac{1}{s} W^{\prime}(s x) \cdot x$ is strictly increasing for $s>0$. Then $\frac{d}{d s} \varphi(s u)>0$ for all $s \in(0,1)$ and $\frac{d}{d s} \varphi(s u)<0$ for all $s \in(1,+\infty)$. So that the path $\lambda u$ is such that $\max _{\lambda>0} \varphi(\lambda u)=\varphi(u)$, and we get $\inf \{\varphi(u): u \in$ $\mathcal{K}\} \geq c$.

Remark 2.5. Lemmas 2.2 and 2.4 imply that any PS sequence of $\varphi$ at a level $b<2 c$ admits a subsequence which, up to translations, converges strongly in $X$ to a critical point $v$ of $\varphi$ such that $\varphi(v)=b$.

By the above remark, the PS sequence given by the mountain pass theorem converges (up to subsequences and translations) to a critical point $\bar{v} \in \mathcal{K}$ such that $\varphi(\bar{v})=c$.

By (h4) (see the proof of lemma 2.4), if we consider the path $\bar{\gamma} \in \Gamma$ defined by $\bar{\gamma}(s)=s s_{0} \bar{v}$, with $s_{0}$ such that $\varphi\left(s_{0} \bar{v}\right)<0$, then it satisfies: 
$\left(\gamma_{1}\right) \max _{s \in[0,1]} \varphi(\bar{\gamma}(s))=\varphi(\bar{v})=c$;

$\left(\gamma_{2}\right) \forall r \in(0, \bar{\rho}) \exists h_{r}>0$ such that if $\gamma(s) \in X \backslash B_{\frac{r}{4}}(\bar{v})$, then $\varphi(\bar{\gamma}(s)) \leq c-2 h_{r}$.

By a cut-off procedure we then obtain:

Lemma 2.6. $\bar{v} \in \mathcal{K}$ is a mountain pass critical point for $\varphi$. In fact, for any $r \in(0, \bar{\rho})$ and $h \in\left(0, h_{r}\right)$ there is a path $\gamma \in \Gamma$ satisfying:

(i) range $\gamma \subset\{\varphi \leq c+h\}$;

(ii) range $\gamma \subset B_{\frac{r}{2}}(\bar{v}) \cup\left\{\varphi \leq c-h_{r}\right\}$;

(iii) supp $\gamma(\theta) \subset[-R, R]$ for any $\theta \in[0,1], R$ being a positive constant independent on $\theta$.

\section{The ASSUmption $\mathcal{W}^{s} \neq \mathcal{W}^{u}$ and RELATED COMPACTNESS PROPERTIES}

By (h1) and (h2), we can characterize the local stable and unstable manifolds as graphs of functions from the configuration to the velocity space.

Lemma 3.1. There exists $\tilde{\delta} \in(0, \bar{\delta})$ such that if we define $\mathcal{W}_{\text {loc }}^{s}=\{(x, p) \in$ $\mathcal{W}^{s}:\left|\Phi^{n}(x, p)\right|<\tilde{\delta}$ for all $\left.n \geq 0\right\}$ and $\mathcal{W}_{l o c}^{u}=\left\{(x, p) \in \mathcal{W}^{u}:\left|\Phi^{n}(x, p)\right|<\right.$ $\tilde{\delta}$ for all $n \leq 0\}$, then there exist $f_{s}, f_{u} \in C^{2}((-\tilde{\delta}, \tilde{\delta}), \mathbb{R})$ such that

$$
\mathcal{W}_{\text {loc }}^{s}=\left\{\left(x, f_{s}(x)\right) ;|x|<\tilde{\delta}\right\}, \quad \mathcal{W}_{\text {loc }}^{u}=\left\{\left(x, f_{u}(x)\right) ;|x|<\tilde{\delta}\right\} .
$$

Proof. By continuous dependence on initial conditions we can fix $\tilde{\delta} \in(0, \bar{\delta})$ such that if $(x, p) \in \mathcal{W}_{l o c}^{s}$ (resp. $\in \mathcal{W}_{\text {loc }}^{u}$ ), then the solution $u$ of (1.1) corresponding to this initial condition verifies $|u(t)| \leq \bar{\delta}$ for any $t \geq 0$ (resp. $t \leq 0$ ).

We define $\varphi_{ \pm}(u)=\frac{1}{2} \int_{\mathbb{R}_{ \pm}}|\dot{u}(t)|^{2}+|u(t)|^{2}-\int_{\mathbb{R}_{ \pm}} \alpha(t) W(u(t)) d t$. By (2.1) we have that $\varphi_{ \pm}$is strictly convex on the convex set $\mathcal{U}_{ \pm, x}=\left\{u \in H^{1}\left(\mathbb{R}_{ \pm}\right), \sup _{t \in \mathbb{R}_{ \pm}}|u(t)| \leq\right.$ $\bar{\delta}, u(0)=x\}$ for any $|x| \leq \bar{\delta}$.

Therefore the minimum problem

$$
\left(P_{ \pm, x}\right) \quad \min \left\{\varphi_{ \pm}(u) ; u \in \mathcal{U}_{ \pm, x}\right\}
$$

admits a unique solution $u_{ \pm, x}$ for any $|x| \leq \bar{\delta}$ which turns out to be the unique solution on $\mathbb{R}_{ \pm}$of (1.1) which verifies the conditions $u_{ \pm, x}(0)=x$ and $\sup _{t \in \mathbb{R}_{ \pm}}\left|u_{ \pm, x}(t)\right| \leq$ $\bar{\delta}$. Since $W \in C^{2}(\mathbb{R})$ it follows that the maps $f_{ \pm}:(-\bar{\delta}, \bar{\delta}) \rightarrow \mathbb{R}, f_{ \pm}(x)=\dot{u}_{ \pm, x}(0)$ are in fact in $C^{2}((-\bar{\delta}, \bar{\delta}))$.

Starting from the local stable and unstable manifolds, it is possible to recover by backward iterations the global stable and unstable manifolds. We have:

$$
\mathcal{W}^{s}=\bigcup_{n \leq 0} \Phi^{n}\left(\mathcal{W}_{\text {loc }}^{s}\right) \quad \text { and } \quad \mathcal{W}^{u}=\bigcup_{n \geq 0} \Phi^{n}\left(\mathcal{W}_{\text {loc }}^{u}\right) .
$$

To study the condition $\mathcal{W}^{u} \neq \mathcal{W}^{s}$ we introduce for $\delta>0$ the functions $T_{\delta}^{ \pm}$: $X \rightarrow[-\infty,+\infty]$ defined by

$$
T_{\delta}^{+}(u)=\sup \{t \in \mathbb{R}:|u(t)|=\delta\} \quad T_{\delta}^{-}(u)=\inf \{t \in \mathbb{R}:|u(t)|=\delta\}
$$

with the agreement that $T_{\delta}^{ \pm}(u)=\mp \infty$ if $\|u\|_{\infty}<\delta$.

Lemma 3.2. If $\mathcal{W}^{u} \neq \mathcal{W}^{s}$, then there exists $\delta_{0} \in\left(0, \min \left\{\tilde{\delta}, \frac{\bar{\delta}}{2}\right\}\right)$ such that

$$
(\#)^{+} 0 \notin\left\{T_{\delta_{0}}^{+}(u): u \in \mathcal{K}\right\} \text { or }(\#)^{-} 0 \notin\left\{T_{\delta_{0}}^{-}(u): u \in \mathcal{K}\right\} \text {. }
$$


Proof. If $\mathcal{W}^{u} \neq \mathcal{W}^{s}$, then by (3.2) at least one of the following cases is verified:

$$
\mathcal{W}_{\text {loc }}^{s} \not \subset \mathcal{W}^{u} \text { or } \mathcal{W}_{\text {loc }}^{u} \not \subset \mathcal{W}^{s} \text {. }
$$

Indeed, if $\mathcal{W}_{\text {loc }}^{s} \subset \mathcal{W}^{u}$, since $\mathcal{W}^{u}$ is $\Phi^{-1}$-invariant, then $\Phi^{n}\left(\mathcal{W}_{\text {loc }}^{s}\right) \subset \mathcal{W}^{u}$ for any $n \leq 0$ and $\mathcal{W}^{s} \subset \mathcal{W}^{u}$ plainly follows by (3.2). Analogously, if $\mathcal{W}_{l o c}^{u} \subset \mathcal{W}^{s}$, then $\mathcal{W}^{u} \subset \mathcal{W}^{s}$

Let us assume that $\mathcal{W}_{\text {loc }}^{s} \not \subset \mathcal{W}^{u}$. Then, there exists $\left|\delta_{0}\right| \leq \min \left\{\tilde{\delta}, \frac{\bar{\delta}}{2}\right\}$ such that $\left(\delta_{0}, f_{s}\left(\delta_{0}\right)\right) \notin \mathcal{W}^{u}$. In addition, by (h5), we have that $\left(-\delta_{0}, f_{s}\left(-\delta_{0}\right)\right) \notin \mathcal{W}^{u}$, hence it is not restrictive to assume $\delta_{0}>0$.

This fact implies that if $u$ is a homoclinic solution, then $T_{\delta_{0}}^{+}(u) \neq 0$. Indeed, if otherwise there exists a homoclinic solution $u$ with $T_{\delta_{0}}^{+}(u)=0$, we have $|u(0)|=\delta_{0}$ and $|u(j)|<\delta_{0}$ for any $j>0$ and hence, since by (h5) we can assume $u(0)=\delta_{0}$, we get $(u(0), \dot{u}(0))=\left(\delta_{0}, f_{s}\left(\delta_{0}\right)\right) \in \mathcal{W}^{u}$, a contradiction. The same argument applies if $\mathcal{W}_{\text {loc }}^{u} \not \subset \mathcal{W}^{s}$ and in this case we get that there exists $\delta_{0} \in\left(0, \min \left\{\tilde{\delta}, \frac{\delta}{2}\right\}\right)$ such that $0 \notin\left\{T_{\delta_{0}}^{-}(u): u \in \mathcal{K}\right\}$.

In the following we assume that $(\#)^{+}$holds, all the arguments being the same if instead $(\#)^{-}$holds. In the following we set $T^{+}(u)=T_{\delta_{0}}^{+}(u)$.

From $(\#)^{+}$we can start applying variational techniques to prove the existence of multibump solutions.

Lemma 3.3. Let $\left(u_{n}\right) \subset X$ be a PS sequence at level $b \in[c, 2 c)$. If the sequence $\left(T^{+}\left(u_{n}\right)\right)$ is a bounded sequence, then there exists $v \in \mathcal{K}$ such that $u_{n} \rightarrow v$ strongly in $X$ and $T^{+}\left(u_{n}\right) \rightarrow T^{+}(v)$ (up to subsequences).

Proof. Since $\left(u_{n}\right)$ is a PS sequence there exists a critical point $v \in \mathcal{K} \cup\{0\}$ such that, up to subsequences, $u_{n} \rightarrow v$ weakly in $X$. In particular $u_{n} \rightarrow v$ in $L_{l o c}^{\infty}(\mathbb{R})$ and since $\left(T^{+}\left(u_{n}\right)\right)$ is bounded there exists $R>0$ such that $\left\|u_{n}\right\|_{L^{\infty}(-R, R)} \geq \delta_{0}$. Therefore we have $v \neq 0$ and since $b \in[c, 2 c)$, by remark 2.5 , we conclude that $u_{n} \rightarrow v$ strongly in $X$.

Moreover, let $T^{+}\left(u_{n_{k}}\right) \rightarrow t^{*}$ as $k \rightarrow+\infty$. The lemma follows if we prove that $t^{*}=T^{+}(v)$.

Since $u_{n} \rightarrow v$ in $L^{\infty}(\mathbb{R}), t^{*} \leq T^{+}(v)$. Arguing by contradiction, assume that $t^{*}<T^{+}(v)$. By continuity there exists $\rho \in\left(0, \frac{1}{2}\left(T^{+}(v)-t^{*}\right)\right)$ such that $|v(t)| \leq \bar{\delta}$ for any $t \in\left[T^{+}(v)-\rho, T^{+}(v)\right]$. Since $\ddot{v}=v-\alpha(t) W^{\prime}(v)$, by (h2), there exists $a>0$ such that $\frac{d}{d t}|v(t)|^{2} \leq-a$ for all $t \in\left[T^{+}(v)-\rho, T^{+}(v)\right]$. Hence we get $\left|v\left(T^{+}(v)-\rho\right)\right|^{2}=\delta_{0}^{2}-\int_{T^{+}(v)-\rho}^{T^{+}(v)} \frac{d}{d t}|v(t)|^{2} d t \geq \delta_{0}^{2}+a \rho$. Hence there exists $\bar{k} \in \mathbb{N}$ such that for any $k \geq \bar{k}$ we have $\left|u_{n_{k}}\left(T^{+}(v)-\rho\right)\right|>\delta_{0}$ and $T^{+}\left(u_{n_{k}}\right)>T^{+}(v)-\rho$, a contradiction.

Let $c^{*} \in(c, 2 c)$, thanks to the above lemma and $(\#)^{+}$we can characterize the critical set $\mathcal{K} \cap\left\{\varphi \leq c^{*}\right\}$ as follows:

Lemma 3.4. There exists $\eta \in\left(0, \frac{1}{2}\right)$ such that if $u \in \mathcal{K} \cap\left\{\varphi \leq c^{*}\right\}$, then $T^{+}(u) \in$ $[j+\eta, j+1-\eta]$ for some $j \in \mathbb{Z}$.

Proof. By periodicity it is sufficient to prove that there exists $\eta \in\left(0, \frac{1}{2}\right)$ for which if $T^{+}(u) \in(-\eta, \eta)$ and $u \in\left\{\varphi \leq c^{*}\right\}$, then $u \notin \mathcal{K}$.

If this is not true there exists a sequence of critical points $\left(u_{n}\right) \subset \mathcal{K} \cap\left\{\varphi \leq c^{*}\right\}$ with $\left|T^{+}\left(u_{n}\right)\right| \leq \frac{1}{n}$.

Then by lemma 3.3 we conclude that $u_{n} \rightarrow v$ strongly in $X$ (up to subsequences) for some $v \in \mathcal{K}$ and $T^{+}(v)=0$, in contradiction with $(\#)^{+}$. 
Let $\bar{v}$ be the mountain pass critical point considered in lemma 2.6 and let $j_{0} \in \mathbb{Z}$ be such that $T^{+}(\bar{v}) \in\left[j_{0}+\eta, j_{0}+1-\eta\right]$. We set

$$
\bar{K}=\left\{u \in \mathcal{K} \cap\left\{\varphi \leq c^{*}\right\} / T^{+}(u) \in\left[j_{0}+\eta, j_{0}+1-\eta\right]\right\} .
$$

By lemma $3.3 \bar{K}$ is a compact subset of $X$. Moreover $\bar{K}$ is an isolated set in $\mathcal{K} \cap\left\{\varphi \leq c^{*}\right\}$. In fact we have

Lemma 3.5. $d\left(\bar{K}, \mathcal{K} \cap\left\{\varphi \leq c^{*}\right\} \backslash \bar{K}\right)=r_{0}>0$.

Proof. If the lemma is not true there exists a sequence $\left(u_{n}\right) \in \bar{K}$ and a sequence $v_{n} \in \mathcal{K} \cap\left\{\varphi \leq c^{*}\right\} \backslash \bar{K}$ such that $\left\|u_{n}-v_{n}\right\| \rightarrow 0$. Since $\bar{K}$ is compact we can assume that $u_{n} \rightarrow v$ with $v \in \bar{K}$. Then $v_{n} \rightarrow v$ and by lemma $3.3 T^{+}\left(v_{n}\right) \rightarrow$ $T^{+}(v) \in\left[j_{0}+\eta, j_{0}+1-\eta\right]$. But since $v_{n} \in \mathcal{K} \cap\left\{\varphi \leq c^{*}\right\} \backslash \bar{K}$, by lemma 3.4 we have $T^{+}\left(v_{n}\right) \in \cup_{j \neq j_{0}}[j+\eta, j+1-\eta]$ for any $n \in \mathbb{N}$, a contradiction which proves the lemma.

Let $\bar{r}=\frac{1}{2} \min \left\{\bar{\rho}, r_{0}\right\}$. By lemmas 2.3 and 3.5 we have

Lemma 3.6. For any $r \in(0, \bar{r})$ there exists $\mu_{r}>0$ such that

$$
\left\|\varphi^{\prime}(u)\right\| \geq \mu_{r} \quad \text { for any } u \in\left(B_{\bar{r}}(\bar{K}) \cap\left\{\varphi \leq c^{*}\right\}\right) \backslash B_{\frac{r}{4}}(\bar{K}) \text {. }
$$

Finally we state a last compactness property which is due to the regularity of the functional $\varphi$.

Lemma 3.7. $\left[0, c^{*}\right] \backslash \varphi(\bar{K})$ is open and dense in $\left[0, c^{*}\right]$.

Proof. Since $\bar{K}$ is compact we have that $\varphi(\bar{K})$ is a closed subset of $\mathbb{R}$. Therefore it is enough to prove that $|\varphi(\bar{K})|=0$.

Since $\varphi \in C^{2}(X, \mathbb{R})$ and $X$ is a real, separable Hilbert space, it is sufficient to prove that $\varphi^{\prime}(u)$ is a (nonlinear) Fredholm operator. Indeed $\operatorname{dim} \operatorname{Ker}\left(\varphi^{\prime \prime}(u)\right) \leq 2$ for any $u \in X$ and we get that $|\varphi(\mathcal{K})|=0$ by applying directly a refined version of the Sard-Smale lemma (see [1], lemma 3.1.47).

We have that $\varphi^{\prime \prime}(u) h=\ddot{h}-h+\alpha(t) W_{q, q}(u(t)) h=L h+\psi(t) h$ where we denote $\psi(t)=\alpha(t) W_{q, q}(u(t))$. Since $L \in L\left(X, H^{-1}(\mathbb{R})\right)$ has bounded inverse, to prove that $\varphi^{\prime \prime}(u)$ is a Fredholm operator, it is sufficient to prove that the multiplication operator $h \rightarrow \psi h$ is compact from $X \rightarrow L^{2}(\mathbb{R})$. This plainly follows since $\psi(t) \rightarrow 0$ as $|t| \rightarrow 0$. Indeed, since $u \in X$ we have that $u(t) \rightarrow 0$ as $|t| \rightarrow \infty$. Therefore, since $\alpha$ is bounded and $W_{q, q}(0)=0$, we obtain $\psi(t) \rightarrow 0$ as $|t| \rightarrow 0$.

Collecting the results obtained above, we have all the ingredients needed to prove existence of multibump solutions. Precisely, we have the existence of a compact set of critical points $\bar{K}$ such that:

(1) Annuli property: For any $r \in(0, \bar{r})$ there exists $\mu_{r}>0$ such that

$$
u \in\left(B_{\bar{r}}(\bar{K}) \cap\left\{\varphi \leq c^{*}\right\}\right) \backslash B_{\frac{r}{4}}(\bar{K}) \Rightarrow\left\|\varphi^{\prime}(u)\right\| \geq \mu_{r} .
$$

(2) Slices property: For any open interval $I \subset\left(0, c^{*}\right), \exists[a, b] \subset I$ and $\exists \tilde{\nu}>0$ such that

$$
u \in B_{\bar{r}}(\bar{K}) \cap\{a \leq \varphi \leq b\} \Rightarrow\left\|\varphi^{\prime}(u)\right\| \geq \tilde{\nu} .
$$

(3) Topological property: For any $r \in(0, \bar{r})$ and $h>0$ there exists $\gamma \in \Gamma$ such that:

(i) range $\gamma \subset\{\varphi \leq c+h\})$;

(ii) range $\gamma \subset B_{\frac{r}{2}}(\bar{K}) \cup\left\{\varphi \leq c-h_{r}\right\}, h_{r}$ given by $\left(\gamma_{2}\right)$; 
(iii) $\operatorname{supp} \gamma(\theta) \subset[-R, R]$ for any $\theta \in[0,1], R$ being a positive constant independent of $\theta$.

These three properties, together with periodicity, are sufficient to apply the Séré multibump construction [18]. For completeness, we give some details in the next section.

\section{Multibump solutions}

To begin let us introduce some notations. For $k, N \in \mathbb{N}$ we set

$$
P(k, N)=\left\{\left(p_{1}, \ldots, p_{k}\right) \in \mathbb{Z}^{k}: p_{i+1}-p_{i} \geq 2 N^{2}+3 N \forall i=1, \ldots, k-1\right\},
$$

and, for $p \in P(k, N)$ we define the intervals:

$$
\begin{array}{cl}
I_{i}=\left(\frac{p_{i-1}+p_{i}}{2}, \frac{p_{i}+p_{i+1}}{2}\right) & (i=1, \ldots, k), \\
M_{i}=\left(p_{i}+N(N+1), p_{i+1}-N(N+1)\right) & (i=0, \ldots, k)
\end{array}
$$

and $M=\bigcup_{i=0}^{k} M_{i}$, with the agreement that $p_{0}=-\infty$ and $p_{k+1}=+\infty$.

Given $\epsilon>0$, let

$$
\mathcal{M}_{\epsilon}=\left\{u \in X:\|u\|_{M_{i}}^{2} \leq \epsilon \forall i=0, \ldots, k\right\} .
$$

In addition, for $p \in P(k, N)$ we introduce the functionals $\varphi_{i}: X \rightarrow \mathbb{R}$ defined by $\varphi_{i}(u)=\frac{1}{2}\|u\|_{I_{i}}^{2}-\int_{I_{i}} \alpha(t) W(u) d t, i=1, \ldots, k$.

We notice that $\varphi=\sum_{i=1}^{k} \varphi_{i}$ and $\varphi_{i} \in C^{1}(X, \mathbb{R})$ with $\varphi_{i}^{\prime}(u) v=\langle u, v\rangle_{I_{i}}-$ $\int_{I_{i}} \alpha(t) W^{\prime}(u) \cdot v d t$ for any $u, v \in X$.

Lastly, given $p \in P(k, N)$, a compact set $K \subset X$ and $r>0$ we set

$$
B_{r}(K ; p)=\left\{u \in X: \inf _{v \in K}\left\|u-v\left(\cdot-p_{i}\right)\right\|_{I_{i}}<r \quad \forall i=1, \ldots, k\right\} .
$$

We point out that $B_{r}(K ; p)$ contains functions with $k$-bumps. In particular, each of these bumps is localized on an interval $I_{i}$, near a $p_{i}$ translated of some point $v \in K$.

For any $r \in(0, \bar{r}), h>0$ we consider the surface $G_{h}: Q=[0,1]^{k} \rightarrow X$ defined by

$$
G_{h}\left(\theta_{1}, \ldots, \theta_{k}\right)=\sum_{i=1}^{k} \gamma\left(\theta_{i}\right)\left(\cdot-p_{i}\right)
$$

where the path $\gamma$ is given by the topological property (3). Note that by the $\mathbb{Z}$ translational invariance, the path translated by $p_{i} \in \mathbb{Z}$ satisfies $(i)-(i i)-(i i i)$ with respect to the translated set $p_{i} * \bar{K}=\left\{u\left(\cdot-p_{i}\right): u \in \bar{K}\right\}$.

If the points $p_{i} \in \mathbb{Z}$ are sufficiently far away one from the others, then the supports of the $\gamma\left(\theta_{i}\right)\left(\cdot-p_{i}\right)(i=1, \ldots, k)$ are disjoint. More precisely, we require $N \geq R, R$ be given by (3)-(iii), so that $\operatorname{supp} \gamma\left(\theta_{i}\right)\left(\cdot-p_{i}\right) \subset I_{i} \backslash M$ and we obtain

$$
\varphi\left(G_{h}(\theta)\right)=\sum_{i=1}^{k} \varphi_{i}\left(G_{h}(\theta)\right)=\sum_{i=1}^{k} \varphi_{i}\left(\gamma\left(\theta_{i}\right)\left(\cdot-p_{i}\right)\right) .
$$

In other words the functional on the surface $G_{h}$ separates into the sum of the truncated functionals on each $\gamma\left(\theta_{i}\right)\left(\cdot-p_{i}\right)$.

Thanks to the annuli and slices properties we can construct for the functional $\varphi$ and each $\varphi_{i}$ a common pseudogradient vector field in $B_{r}(\bar{K} ; p)$ to get a deformation of the surface $G_{h}(Q)$. 
The existence and the properties of this pseudogradient are stated in the following lemma (see [13] for a proof).

Lemma 4.1. Let $r \in(0, \bar{r})$ and $r_{1}<r_{2}<r_{3}$, with $r_{1}, r_{2}, r_{3} \in\left(\frac{2}{3} r, \frac{5}{6} r\right)$. There exists $\mu=\mu\left(r, r_{1}, r_{3}\right)>0$ such that given $h \in\left(0, h_{r}\right)$, there are two intervals $\left[c_{-}-\lambda, c_{-}+2 \lambda\right] \subset\left(c-h, c-\frac{h}{2}\right),\left[c_{+}-\lambda, c_{+}+2 \lambda\right] \subset\left(c+\frac{3 h}{2}, c+2 h\right)$ and a real number $\epsilon_{1}>0$ such that:

$\forall \epsilon \in\left(0, \epsilon_{1}\right)$ there exists $N_{0} \in \mathbb{N}$ for which for any $k \in \mathbb{N}$ and $p \in P\left(k, N_{0}\right)$, there exists a locally Lipschitz continuous function $\mathcal{W}: X \rightarrow X$ which verifies

$(\mathcal{W} 1) \max _{1 \leq j \leq k}\|\mathcal{W}(u)\|_{I_{j}} \leq 1, \varphi^{\prime}(u) \mathcal{W}(u) \geq 0 \forall u \in X, \mathcal{W}(u)=0 \forall u \in X \backslash$ $B_{r_{3}}(\bar{K} ; p)$

$(\mathcal{W} 2) \varphi_{i}^{\prime}(u) \mathcal{W}(u) \geq \mu$ if $r_{1} \leq \inf _{v \in \bar{K}}\left\|u-v\left(\cdot-p_{i}\right)\right\|_{I_{i}} \leq r_{2}, u \in B_{r_{2}}(\bar{K} ; p) \cap\left\{\varphi_{i} \leq\right.$ $\left.c_{+}\right\}$

$(\mathcal{W} 3) \varphi_{i}(u) \mathcal{W}(u) \geq 0 \forall u \in\left\{c_{+} \leq \varphi_{i} \leq c_{+}+\lambda\right\} \cup\left\{c_{-} \leq \varphi_{i} \leq c_{-}+\lambda\right\}$;

$(\mathcal{W} 4)\langle u, \mathcal{W}(u)\rangle_{M_{j}} \geq 0 \forall j \in\{0, \ldots, k\}$ if $u \in X \backslash \mathcal{M}_{\epsilon}$.

Moreover if $K \cap B_{r_{3}}(\bar{K} ; p)=\emptyset$, then there exists $\mu_{p}>0$ such that

$(\mathcal{W} 5) \varphi^{\prime}(u) \mathcal{W}(u) \geq \mu_{p} \forall u \in B_{r_{2}}(\bar{K} ; p)$.

Then we have

Theorem 4.2. Let (h1)-(h5) and (\#) hold. Then, for any $r>0$ there exists $N_{r} \in \mathbb{N}$ such that for any $k \in \mathbb{N}$ and $p \in P\left(k, N_{r}\right)$ we have $B_{r}(\bar{K} ; p) \cap \mathcal{K} \neq \emptyset$.

Proof. Let $r \in\left(0, \frac{\bar{r}}{4}\right)$ and $r_{1}<r_{2}<r_{3}$, with $r_{1}, r_{2}, r_{3} \in\left(\frac{2}{3} r, \frac{5}{6} r\right)$. We set $\Delta=$ $\frac{\mu\left(r_{2}-r_{1}\right)}{4}$, where $\mu$ is given by lemma 4.1. We fix $h \leq \frac{\Delta}{16}$ and we put $G=G_{h}$. Let $0<$ $\epsilon<\frac{1}{4} \min \left\{\epsilon_{1}, r^{2}-r_{3}^{2}, c-c_{-}\right\}$and $N>\max \left\{N_{0}, R\right\}$ be $\operatorname{such}$ that $\sup _{v \in \bar{K}}\|v\|_{|t| \geq N}^{2} \leq$ $\epsilon$, where $N_{0}, \epsilon_{1}, c_{-}$are given by lemma 4.1 and $R$ by (3)-(iii) relatively to the value of $r, r_{1}, r_{2}, r_{3}, h$ fixed above.

Arguing by contradiction we can assume that for the value of $r$ fixed above there exist $k \in \mathbb{N}$ and $p \in P(k, N)$ such that $B_{r}(\bar{K} ; p) \cap \mathcal{K}=\emptyset$. By lemma 4.1 there exists a vector field $\mathcal{W}$ satisfying the properties $(\mathcal{W} 1)-(\mathcal{W} 5)$ and we consider the Cauchy problem

$$
\left\{\begin{array}{l}
\frac{d \eta}{d s}=-\mathcal{W}(\eta), \\
\eta(0, u)=u
\end{array}\right.
$$

Since $\mathcal{W}$ is a bounded locally Lipschitz continuous vector field we have that for any $u \in X$ there exists a unique solution $\eta(\cdot, u) \in C\left(\mathbb{R}^{+}, X\right)$, depending continuously on $u \in X$.

Note that we have $G(\partial Q) \subseteq X \backslash B_{r_{3}}(\bar{K} ; p)$ and by $(\mathcal{W} 1)$ we get

$$
\eta(s, G(\theta))=G(\theta) \quad \forall \theta \in \partial Q \forall s \in \mathbb{R}^{+} .
$$

Moreover, for any $\theta$ for which $G(\theta) \in X \backslash B_{r_{1}}(\bar{K} ; p)$ there exists $i=i(\theta)$ such that $\eta(s, G(\theta)) \in\left\{\varphi_{i} \leq c_{-}\right\}$for any $s \in \mathbb{R}^{+}$. Indeed, if $G(\theta) \in X \backslash B_{r_{1}}(\bar{K} ; p)$, then there exists $i=i(\theta)$ for which $\inf _{v \in \bar{K}}\left\|p_{i} * \gamma\left(\theta_{i}\right)-p_{i} * v\right\|^{2} \geq \inf _{v \in \bar{K}}\left\|p_{i} * \gamma\left(\theta_{i}\right)-p_{i} * v\right\|_{I_{i}}^{2}>r_{1}^{2}$

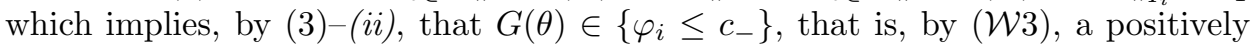
invariant set. 
Then, since $\varphi$ sends bounded sets into bounded sets we get that there exists $\tau>0$ for which $\forall u \in B_{r_{1}}(\bar{K} ; p)$ there exists $\bar{s} \in(0, \tau]$ such that $\eta(\bar{s}, u) \notin B_{r_{2}}(\bar{K} ; p)$. Indeed, if not, setting $\sigma=\sup _{v, w \in B_{r_{2}}(\bar{K} ; p)}|\varphi(v)-\varphi(w)|$ and taking $\tau \geq \frac{2 \sigma}{\mu_{p}}$, by $(\mathcal{W} 5)$, we get

$$
\sigma \geq|\varphi(\eta(\tau, u))-\varphi(u)|=\int_{0}^{\tau} \varphi^{\prime}(\eta(s, u)) \mathcal{W}(\eta(s, u)) \geq \tau \mu_{p} \geq 2 \sigma,
$$

a contradiction.

Hence, for any $u \in B_{r_{1}}(\bar{K} ; p)$ there is is an index $i=i(\theta)$ and an interval $\left[s_{1}, s_{2}\right] \subset$ $(0, \tau]$ such that $\inf _{v \in p_{i} * \bar{K}}\left\|\eta\left(s_{1}, u\right)-v\right\|_{I_{i}}=r_{1}, \inf _{v \in p_{i} * \bar{K}}\left\|\eta\left(s_{2}, u\right)-v\right\|_{I_{i}}=r_{2}$ and $r_{1} \leq \inf _{v \in p_{i} * \bar{K}}\|\eta(s, u)-v\|_{I_{i}} \leq r_{2}$ for any $s \in\left(s_{1}, s_{2}\right)$. Then, by $(\mathcal{W} 2)$, for any $\theta \in Q$ for which $G(\theta) \in B_{r_{1}}(\bar{K} ; p)$, we have $\varphi_{i}\left(\eta\left(s_{2}, G(\theta)\right)\right) \leq \varphi_{i}\left(\eta\left(s_{1}, G(\theta)\right)\right)-2 \Delta$. Since by construction $G(\theta) \in\left\{\varphi_{i} \leq c_{+}\right\}$and since, by $(\mathcal{W} 3),\left\{\varphi_{i} \leq c_{+}\right\}$is positively invariant, we get $\varphi_{i}\left(\eta\left(s_{2}, G(\theta)\right)\right) \leq c_{+}-2 \Delta<c_{-}$. Therefore $\varphi_{i}(\eta(\tau, G(\theta))) \leq c_{-}$ follows from the positive invariance of $\left\{\varphi_{i} \leq c_{-}\right\}$given by $(\mathcal{W} 3)$.

Collecting the results and setting $\bar{G}(\theta)=\eta(\tau, G(\theta))$, we obtain

$$
\forall \theta \in Q, \exists i \in\{1, \ldots, k\} / \varphi_{i}(\bar{G}(\theta)) \leq c_{-} .
$$

Thanks to this last property we can select on $Q$ a path $\xi$ joining two opposite faces $\left\{\theta_{i}=0\right\}$ and $\left\{\theta_{i}=1\right\}$ along which the function $\varphi_{i} \circ \bar{G}$ takes values strictly less than $c$ for some $i \in\{1, \ldots, k\}$. Precisely:

$$
\begin{aligned}
& \text { there exists } i \in\{1, \ldots, k\} \text { and } \xi \in C([0,1], Q) \text { such that } \\
& \xi(0) \in\left\{\theta_{i}=0\right\}, \xi(1) \in\left\{\theta_{i}=1\right\} \text { and } \varphi_{i}(\bar{G}(\theta))<c_{-}+\epsilon, \\
& \text { for any } \theta \in \text { range } \xi \text {. }
\end{aligned}
$$

Indeed, assuming the contrary, the set $D_{i}=\left\{\theta \in Q: \varphi_{i}(\bar{G}(\theta)) \geq c_{-}+\epsilon\right\}$ for any $i \in\{1, \ldots, k\}$ separates in $Q$ the faces $F_{i}^{0}=\left\{\theta_{i}=0\right\}$ and $F_{i}^{1}=\left\{\theta_{i}=1\right\}$. For any $i \in\{1, \ldots, k\}$ let $C_{i}$ be the component of $Q \backslash D_{i}$ which contains the face $F_{i}^{1}$ and let us define a function $f_{i}: Q \rightarrow \mathbb{R}$ as follows:

$$
f_{i}(\theta)= \begin{cases}\operatorname{dist}\left(\theta, D_{i}\right) & \text { if } \theta \in Q \backslash C_{i}, \\ -\operatorname{dist}\left(\theta, D_{i}\right) & \text { if } \theta \in C_{i} .\end{cases}
$$

Then, $f_{i} \in C(Q, \mathbb{R}),\left.f_{i}\right|_{F_{i}^{0}} \geq 0,\left.f_{i}\right|_{F_{i}^{1}} \leq 0$ and $f_{i}(\theta)=0$ if and only if $\theta \in D_{i}$. Using a Miranda fixed point theorem ([10]), we get that there exists $\theta \in Q$ such that $f_{i}(\theta)=0$ for all $i \in\{1, \ldots, k\}$, hence $\bigcap_{i} D_{i} \neq \emptyset$, which is in contradiction with the property (4.2).

Note also that, by $(\mathcal{W} 4)$, the set $\mathcal{M}_{\epsilon}$ is positively invariant and since, by (3)-(iii) and the choice of $N, G(Q) \subseteq \mathcal{M}_{\epsilon}$, we have

$$
\eta(s, G(Q)) \subseteq \mathcal{M}_{\epsilon} \quad \forall s \in \mathbb{R}^{+} .
$$

Finally, thanks to (4.1), (4.3) and (4.4) we get a contradiction. Indeed, let $\chi \in C^{\infty}(\mathbb{R},[0,1])$ with $\sup _{t \in \mathbb{R}}|\dot{\chi}(t)| \leq 1$ be such that $\chi(t)=1$ if $t \in I_{i} \backslash M$ and $\chi(t)=0$ if $t \in \mathbb{R} \backslash I_{i}$, where $i \in\{1, \ldots, k\}$ is the index given by (4.3). Notice that $\|\chi u\|_{I_{i} \cap M}^{2} \leq 3\|u\|_{I_{i} \cap M}^{2}$ and $\|(1-\chi) u\|_{I_{i} \cap M}^{2} \leq 3\|u\|_{I_{i} \cap M}^{2}$ for any $u \in X$. Then, we define a path $g:[0,1] \rightarrow X$ by setting $g(s)=\chi \bar{G}(\xi(s))$ for $s \in[0,1]$. By $(4.1)$ and 
(3)-(iii) we have that

$$
g(0)=0 \quad \text { and } g(1)=\gamma(1)\left(\cdot-p_{i}\right)
$$

and $g \in \Gamma$. To get the contradiction, we show that $\varphi(g(s))<c$ for any $s \in[0,1]$. Indeed, since $\bar{G}(\xi(s)) \in \mathcal{M}_{\epsilon}$ and $\epsilon<\frac{c-c_{-}}{4}$, we get

$$
\begin{aligned}
\varphi(g(s)) & =\varphi_{i}(g(s)) \leq \varphi_{i}(\bar{G}(\xi(s)))+\frac{1}{2}\|g(s)\|_{I_{i} \cap M}^{2} \\
& +\int_{I_{i} \cap M} \alpha(t)(W(\bar{G}(\xi(s)))-W(g(s))) d t \leq c_{-}+4 \epsilon<c .
\end{aligned}
$$

Finally we note that since the minimum distance $N$ between two adjacent bumps does not depend on the number of bumps, we can consider the $C_{l o c}^{1}$ closure of the set of multibump homoclinic solutions given by theorem 4.2 and we obtain the complete result.

\section{REFERENCES}

1. M.S. Berger, Nonlinearity and Functional Analysis. Lectures on Nonlinear Problems in Mathematical Analysis, Academic Press, 1977. MR 58:7671

2. U. Bessi, A Variational Proof of a Sitnikov-like Theorem, Nonlinear Anal. 20 (1993), 13031318. MR 94e:58020

3. S.V. Bolotin, Existence of homoclinic motions, Vestnik Moskov. Univ. Ser. I Mat. Mekh. 6 (1980), 98-103. MR 85j:58051

4. B. Buffoni and E. Séré, A global condition for quasi-random behavior in a class of conservative systems, Comm. Pure Appl. Math. 49 (1996), 285-305. MR 97g:58033

5. S.N. Chow, J. Hale and J. Mallet-Paret, An example of bifurcation to homoclinic orbits, J. Diff. Equat. 37 (1980), 351-373. MR 81m:58056

6. K. Cieliebak and E. Séré, Pseudo-holomorphic curves and the shadowing lemma, Duke Math. J. (to appear).

7. V. Coti Zelati, I. Ekeland and E. Séré, A variational approach to homoclinic orbits in Hamiltonian systems, Math.Ann. 288 (1990), 133-160. MR 91g:58065

8. V. Coti Zelati and P.H. Rabinowitz, Homoclinic orbits for second order Hamiltonian systems possessing superquadratic potentials, J. Amer. Math. Soc. 4 (1991), 693-727. MR 93e:58023

9. J.L. Lions, The concentration-compactness principle in the calculus of variations. The locally compact case, I, Ann. Inst. H. Poincaré Anal. Nonlin. 1 (1984), 109-145. MR 87e:49035a

10. C. Miranda, Un'osservazione su un teorema di Brouwer, Boll. Unione Mat. Ital. 3 (1940), 5-7. MR 3:60b

11. P. Montecchiari, M. Nolasco and S. Terracini, Multiplicity of homoclinics for a class of time recurrent second order Hamiltonian systems, Calc. of Var. (to appear). CMP 98:02

12. J. Moser, Stable and random motions in dynamical systems, Princeton University Press, 1973. MR 56:1355

13. M. Nolasco, Multibump solutions for a class of time dependent second order Hamiltonian systems, Ph.D. thesis, SISSA, 1995.

14. K.J. Palmer, Exponential dichotomies and transversal homoclinic points, J. Diff. Equat. 55 (1984), 222-256. MR 86d:58088

15. M. Pollicott, Lectures on ergodic theory and Pesin theory on compact manifolds, London Math. Soc., Lec. Note Series, vol. 180, Cambridge Univ. Press, 1983. MR 94k:58080

16. P.H. Rabinowitz, A multibump construction in a degenerate setting, Calc. Var. Partial Differential Equations 5 (1997), 159-182. MR 98b:58034

17. E. Séré, Existence of infinitely many homoclinic orbits in Hamiltonian systems, Math. Z. 209 (1992), 27-42. MR 92k:58201

18. E. Séré, Looking for the Bernoulli shift, Ann. Inst. H. Poincaré, Anal. Non Linéaire 10 (1993), 561-590. MR 95b:58031 
3724 PIERO MONTECCHIARI, MARGHERITA NOLASCO, AND SUSANNA TERRACINI

19. S. Smale, Differentiable dynamical systems, Bull. Amer. Math. Soc. 73 (1967), 747-817. MR 37:3598

20. S. Wiggins, Global bifurcations and chaos, Applied Mathematical Sciences, vol. 73, SpringerVerlag, 1988. MR 89m:58057

Dipartimento di Matematica, Universitá degli studi di Trieste, Piazzale Europa 1 , 34013 Trieste, Italy

E-mail address: montec@univ.trieste.it

S.I.S.S.A., via Beirut 4, 34013 Trieste, Italy

E-mail address: nolasco@neumann.sissa.it

Dipartimento di Matematica del Politecnico, Piazza Leonardo da Vinci 32, 20133 Milano, Italy

E-mail address: suster@ipmma1.mate.polimi.it 